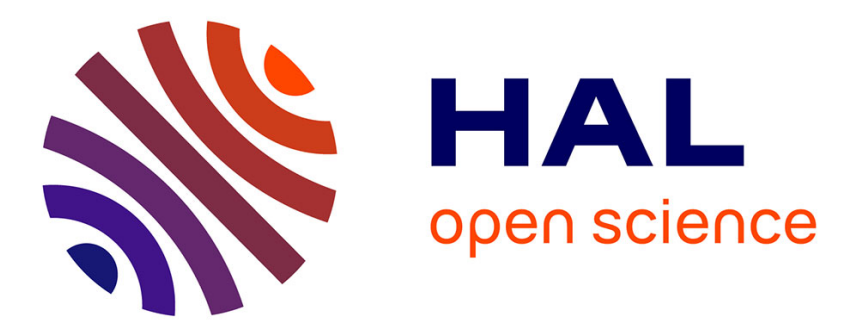

\title{
Mesostructure evolution of cement pastes with addition of superplasticizers highlighted by dispersion indices
}

\author{
Caroline Autier, Nathalie Azema, Jean-Marie Taulemesse, Laurent Clerc
}

\section{To cite this version:}

Caroline Autier, Nathalie Azema, Jean-Marie Taulemesse, Laurent Clerc. Mesostructure evolution of cement pastes with addition of superplasticizers highlighted by dispersion indices. Powder Technology, 2013, 249, pp.282-289. 10.1016/j.powtec.2013.08.036 . hal-02949485

\section{HAL Id: hal-02949485 \\ https://hal.science/hal-02949485}

Submitted on 25 Mar 2021

HAL is a multi-disciplinary open access archive for the deposit and dissemination of scientific research documents, whether they are published or not. The documents may come from teaching and research institutions in France or abroad, or from public or private research centers.
L'archive ouverte pluridisciplinaire HAL, est destinée au dépôt et à la diffusion de documents scientifiques de niveau recherche, publiés ou non, émanant des établissements d'enseignement et de recherche français ou étrangers, des laboratoires publics ou privés. 


\title{
Mesostructure evolution of cement pastes with addition of superplasticizers highlighted by dispersion indices
}

\author{
Caroline Autier, Nathalie Azema *, Jean-Marie Taulemesse, Laurent Clerc \\ Centre des matériaux de l'Ecole des Mines d'Alès, 6 avenue de Clavières, 30319 Alès cedex, France
}

\section{A B S T R A C T}

One of the parameters that condition the workability of concrete is the dispersion state of cement paste. Water trapped into the agglomerates does not contribute to flow properties and fine particles forming agglomerates are not available for packing optimization. Therefore, superplasticizers have been developed to disperse particles, thus increasing the flowability of the material for the same water/cement ratio. Using the complementarity between scanning electron microscopy (SEM) and laser granulometry, dispersion states of cement pastes were characterized. The different kinds of particles and their mesostructural organization were identified, along with transfers of particles that can occur between the different granulometric classes. For that purpose, several liquid media were used for the analysis and different superplasticizers were incorporated for the mixing. Disper-sion indices were developed and compared with the flow behavior of pastes. This comparison underlines the link between mesoscopic state and rheological behavior. This multi-scale approach of experimental characterization may be easily applied to other studies characterizing the influence of surfactants on dispersion phenomena in several fundamental and industrial areas.

Keywords:

Granular characteristics

Dispersion index

Superplasticizers

Cement paste

Rheology

\section{Introduction}

Packing and flow properties of mortars and concretes control workability in the fresh state along with the strength and durability of these materials at hardened state [1]. Both parameters are strongly dependent on the dispersion state of cement paste composed of water, cement, mineral additives and admixtures. More precisely, the dispersion state impacts concrete workability through trapping of water in the agglomerates which does not contribute to flow properties [2]. Furthermore, fine particles involved in the agglomerates are not available anymore to increase maximum packing [1]. Superplasticizers (like polycarboxylate or lignosulfonate) have been developed for years in order to increase particle dispersion, thus improving the rheological properties of the material [3,4].

Many studies correlate the superplasticizer adsorption on mineral particles with the rheological behavior of the suspension [5-8]. However, cement pastes are very heterogeneous systems by their chemical and crystallographic composition and their granular characteristics. Moreover, cement particles are chemically reactive with water, thus increasing heterogeneity and leading to an evolution of the particles characteristics. These are the reasons why many studies were conducted on pure synthetic phases, inert model systems, like magnesium oxide $[9,10]$, or by modeling $[11,12]$. Granular aspects and their influence on materials properties are poorly researched [13-18] and only a few

\footnotetext{
* Corresponding author.

E-mail address: nathalie.azema@mines-ales.fr (N. Azema).
}

studies developed a morpho-granulometric approach based on granulometry and scanning electron microscopy techniques [19-21]. They mainly focused on particle shape and surface, and the characterization of the dispersion/agglomeration phenomena by identifying the different granular populations and associated transfers of particles was studied qualitatively $[22,23]$.

A recent review of J. Stark [24] on cement hydration and microstructure analysis confirm that a high number of studies are connected to cement hydration. Those concerning microstructure are focused on hardened cement paste, mortar or concrete and not on cement paste during dormant period.

The aim of this work is to have a better understanding of the granular and the physicochemical phenomena that occur during this slow chemical reaction period, without taking an interest in chemical characterization of the different cement phases which have been particularly well studied. In this objective, we propose to explore the mesostructural scale of these systems and their evolution, with all its complexity, by working on Ordinary Portland Cement (OPC) pastes during dormant period when the chemical reactivity is slow $[24,25]$. In these conditions, the study of physicochemical phenomena is favored over hydration reactions.

As a first step, different granulometric populations were identified in a unadmixtured paste, using the complementarity of scanning electron microscopy (SEM) and laser granulometry.

With the object to make a complete identification and designated an appropriate liquid as suspended medium, different granular characteristics of particles and agglomerates were characterized using several media like air, deionized water, ethyl alcohol and synthetic pore solution. 
In addition, several dispersion states of the paste were also generated using different amounts of polycarboxylate-based superplasticizers of different structures in order to underline the evolution of the particle size distribution, reflecting the meso-structural organization [26].

According to these results, dispersion indices were introduced and related with the flow behavior of the corresponding pastes. Our aim was to establish a relationship between the evolution of the mesostructural organization of a fresh cement paste and its rheological behavior. The dispersion indices should allow us to have a better understanding of the influence of superplasticizers (amount, molecular structure...) on rheological behavior of a cement pastes. This multiscale approach of experimental characterization may be easily applied to other studies characterizing the influence of surfactants on dispersion phenomena in several fundamental and industrial areas.

\section{Material and methods}

\subsection{Materials}

An Ordinary Portland Cement (OPC) of class CEM I 52.5 N provided from Calcia of which chemical and granular characteristics are presented in Table 1 was used for this study. The oxides proportions were determined by X-ray fluorescence. The different specific area characterizations (namely Blaine fineness and specific surface areas by laser granulometry) show similar values about $0.4 \mathrm{~m}^{2} / \mathrm{g}$, and a B.E.T specific surface area value equals to $1 \mathrm{~m}^{2} / \mathrm{g}$. Therefore, the chosen cement particles show no area porosity, and a few agglomerates in the powdery state.

The polycarboxylate-based superplasticizers were provided by CHRYSO Company. A generalized chemical structure is shown in Fig. 1. The molecule is a polymer of methacrylic acid partially esterified with methoxy-terminated polyoxyethylene groups (PEO). It is believed that adsorption occurs via the carboxylic acid groups and the dispersion is given by the PEO grafted chains because the unfolding of the PEO in the pore solution generates steric repulsion. The efficiency of polycarboxylates is dependent on the side chain length and the grafting ratio on the backbone [27-29]. The four polycarboxylates studied are called AA, A, B, and C later on this paper (Fig. 1).

The ester grafting ratio $(\mathrm{m} / \mathrm{n})$ increases from $10 \%$ to $40 \%$ between the PCE AA and C while PEO length (p) is fixed.

\subsection{Paste preparation}

As shown in Fig. 2, the mixing equipment consists in a twisted mixing anchor blade adapted to a Stuart, SS 30 mixer and a stainless steel beaker. The cement suspensions were prepared by adding the powder to deionized water and admixture in the beaker, and then by mixing at a speed of $185 \mathrm{rpm}$ during $20 \mathrm{~min}$ in order to work during the dormant period (this period lasts two hours for the cement studied). Pastes were kept under stirring throughout the analysis. Cement suspensions were prepared with $200 \mathrm{~g}$ of cement and a water to cement ratio $(\mathrm{w} / \mathrm{c})$ of 0.5 (including the amount of water provided by the admixture).

This ratio was imposed by the mixing equipment which is not powerful enough to mix unadmixtured paste with lower w/c. The ratio used

Table 1

Different specific surfaces of the cement powder.

\begin{tabular}{ll}
\hline Cement characteristic & Value \\
\hline B.E.T. specific surface area & $1.1 \mathrm{~m}^{2} / \mathrm{g}$ \\
Blaine specific surface & $0.4 \mathrm{~m}^{2} / \mathrm{g}$ \\
Specific surface area by granulometry & $0.3 \mathrm{~m}^{2} / \mathrm{g}$ \\
& $0.4 \mathrm{~m}^{2} / \mathrm{g}$ \\
- Deionized water & \\
- Dry way & \\
\hline
\end{tabular}

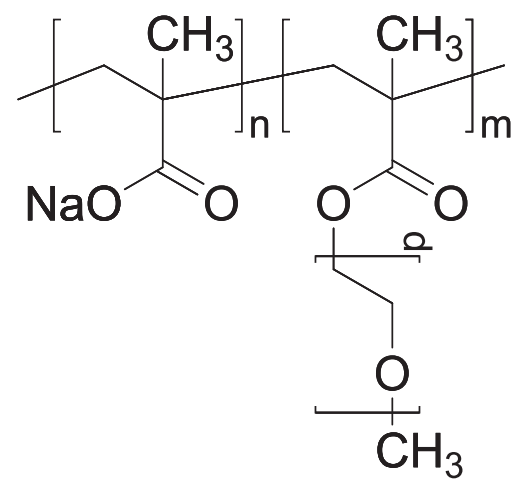

Fig. 1. Chemical structure of a polycarboxylate.

is closer to self-compacting concrete (SCC) applications where superplasticizers are also widely used than high-performance concrete (HPC). Each polycarboxylate was tested with weight dosages of $0.025 \%, 0.05 \%, 0.08 \%, 0.1 \%$ and $1 \%$ of dry active matter with respect to cement weight. These ratios cover the ratios used in the concrete industry.

\subsection{Granular characterization}

Granulometric characterization of powder and pastes was performed using a LS 230 laser granulometer (Beckman Coulter) of which analytic range extends from $40 \mathrm{~nm}$ to $2 \mathrm{~mm}$. The differential particle size distribution of the pastes and of the cement powder was investigated in deionized water, pure ethyl alcohol, a synthetic pore solution and in air, in order to study the influence of the media. We can note that the pore solution used is a salt aqueous solution containing the main ions present in the liquid phase of the cement paste (gypsum: 2.1 g.L $\mathrm{L}^{-1}$, potassium sulfate: 8.7 g.L $\mathrm{L}^{-1}$, sodium hydroxide ( $50 \%$ solution): 14.1 g.L ${ }^{-1}$ ). The optical model used for cement materials has been computed for a refractive index with a real part of 1.7 and an imaginary part of 1 . The analysis time was $1 \mathrm{~min}$ for all the media used and the speed of pump is reduced to the minimum in order not to destroy the agglomerates. A previous study allowed checking that the analysis time is much lower than the dissolution rate of the cement in deionized water for diluted suspensions. Therefore, the adequacy of this liquid media to the study of the admixture influence was checked (Section 3.2). The particle size distributions obtained were expressed in a percent by volume and are normalized to $100 \%$.

The granular characterization was completed with a microscopic analysis performed using a high resolution environmental electronic microscope Quanta 200 FEG from FEI Company. A specific sample preparation technique was developed in order to identify the different granular kinds of particles and their agglomeration state. Pastes were

\section{Stirring speed $185 \mathrm{rpm}$}

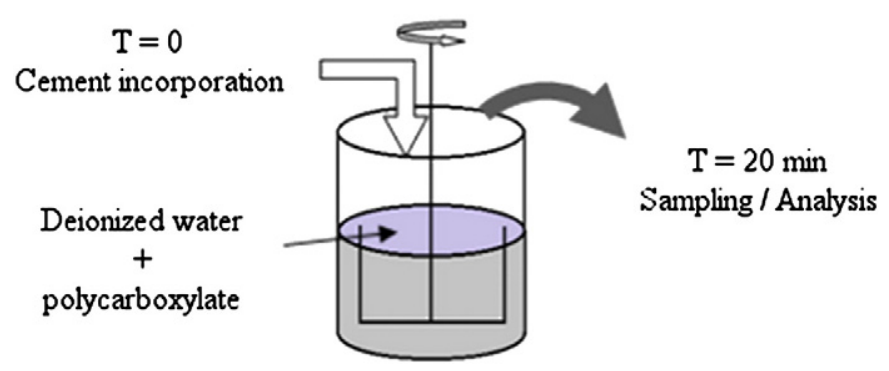

Fig. 2. Procedure of implementation of the cement pastes. 
diluted at about $1 \%$ weight in a liquid (deionized water or ethyl alcohol) and submitted to a low magnetic stirring (in order to preserve the agglomerates) during $1 \mathrm{~min}$. A drop of this suspension was deposited on a liquid drop which was already disposed on a glass slide. The excess water is absorbed which causes a gradient of particle sizes, without destroying the agglomerates formed. The finest particles and agglomerates move toward the more dilute area, whereas the larger remain localized in the concentrated area.

We can remark that the advantage of this preparation method is allowing a distinction between the individual particles and the agglomerates formed. Then these microscopic observations will be more easily compared to granular characteristics obtained by laser granulometry. However, no difference has been noted by SEM observations in the agglomeration states of pastes due to superplasticizer concentrations.

\subsection{Fluidity of cement paste}

The rheological behavior of the pastes was characterized using a stress-controlled shear rheometer (AR 2000, TA Instrument). In order to minimize sedimentation effects on the rheological measurement, vane geometry was chosen (diameter $28 \mathrm{~mm}$, height $42 \mathrm{~mm}$ ). The temperature was fixed to $25{ }^{\circ} \mathrm{C}$. A pre-shear at $50 \mathrm{~s}^{-1}$ was performed during $30 \mathrm{~s}$ before the measurement. Then, steady state flow steps were performed from $5 \mathrm{~s}^{-1}$ to $125 \mathrm{~s}^{-1}$. The rheology of cement-based materials was described by the yield stress determined with the Bingham model applied between $30 \mathrm{~s}^{-1}$ and $125 \mathrm{~s}^{-1}$. Each formulation of paste was analyzed on five different samples.

\section{Results and discussions}

\subsection{Identification of granular populations in unadmixtured paste}

Particle size distributions of the cement powder and cement pastes in different liquid media are shown in Fig. 3. These systems are very polydisperse. Indeed, their size ranges from sub-micronic dimensions (finest particles observed by SEM show a diameter about 0.1-0.2 $\mu \mathrm{m}$; $50-60 \mathrm{~nm}$ by laser granulometry) to a maximum size of around $200 \mu \mathrm{m}$.

Moreover, the particle surface in SEM micrographs appears smooth and without roughness in good agreement with the low values of specific surfaces measured.
Note that ethyl alcohol appears to be a dispersant medium (usually used in industrial control), in contrast to aqueous media (deionized water and pore solution almost similar) and analysis performed by the dry way. Indeed, the main mode is around $25 \mu \mathrm{m}$ in aqueous media and in air, whereas it is only around $10 \mu \mathrm{m}$ in the alcohol medium (Fig. 3-Table 2).

The comparison between the maximum diameter on the particle size distributions in ethyl alcoholic medium of powder ( $40 \mu \mathrm{m})$ and paste $(60 \mu \mathrm{m})$ allows underlining a slight attrition phenomenon during the implementation of the paste. Indeed, ethyl alcohol is a dispersant medium, this dispersion concerns unit particles. Microscopy observations performed on the same samples confirm this hypothesis. Moreover the cement paste analyzed in alcohol media shows an important submicronic mode resulting of particle dispersion or hydrate precipitation (acicular particles that have been only observed in alcohol at this age of the paste). The submicronic particles can originate from those already existing in the granulometric distribution of powder and those that may be produced by attrition during paste preparation. Table 2 shows that deionized water seems to be an interesting suspending fluid for studying the influence of superplasticizers on the mesostructure because of its initial agglomerating properties.

The initial unit particles of cement show a modular and angular shape, whereas hydrates show a specific (cubic or acicular with a shape factor length/diameter of 2) crystalline shape (Fig. 4c). The surface of the particles appears smooth according to the low values of specific surfaces. As we mentioned in the introduction, the identification of the crystalline phases was not investigated in this work.

Agglomerates present in the dry powder correspond to the association of sub-micronic particles or micronic particles on particles of several tens or even hundreds of microns (Fig. 4a). Their dispersion would affect only slightly the size distribution for particles greater than the mode. In this study, the agglomerate is defined as the association of (unit or agglomerated) particles linked together by weak physical bonds (Van der Waals forces).

Cement paste analyzed in aqueous medium allows observing another agglomeration phenomenon corresponding to the association of particles, smaller than the main mode, associated with each other (Fig. 4b).

Furthermore, the particle size distribution is similar to the distribution of the powder to which are added new cubic crystalline phases around $1 \mu \mathrm{m}$. These phases are characteristic of hydration products, like the acicular hydration products of length $0.3-0.7 \mu \mathrm{m}$ located on the particle surface, which are well dispersed in ethyl alcohol (Fig. 4c).

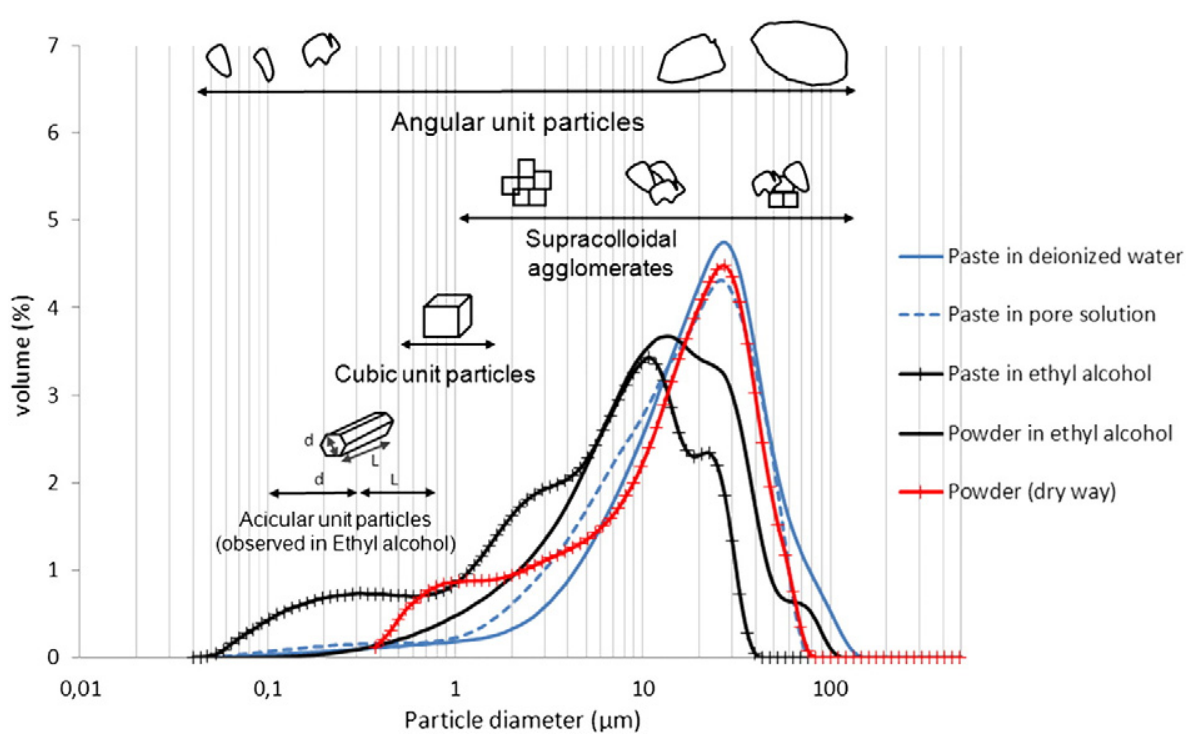

Fig. 3. Distributions of powder and paste analyzed in different media. 
Table 2

Granulometric characteristics of powder and paste analyzed in different media.

\begin{tabular}{|c|c|c|c|c|c|}
\hline & \multicolumn{2}{|c|}{ Main mode $(\mu \mathrm{m})$} & \multicolumn{2}{|c|}{$\begin{array}{l}\text { Spreading }\left(d_{98}-d_{0,1}\right) \\
(\mu \mathrm{m})\end{array}$} & \multirow[t]{2}{*}{$\begin{array}{l}\text { Number } \\
\text { of samples }\end{array}$} \\
\hline & Average & $\begin{array}{l}\text { Standard } \\
\text { deviation }\end{array}$ & Average & $\begin{array}{l}\text { Standard } \\
\text { deviation }\end{array}$ & \\
\hline Powder (dry way) & 26.6 & 1.3 & 64.9 & 19.1 & 6 \\
\hline Paste in deionized water & 24.6 & 1.9 & 92.9 & 11.8 & 5 \\
\hline Paste in pore solution & 23.9 & 2.1 & 61.0 & 5.6 & 4 \\
\hline Paste in ethyl alcohol & 11.9 & 1.6 & 33.3 & 5.7 & 4 \\
\hline Powder in ethyl alcohol & 15.0 & 1.5 & 47.0 & 12.6 & 4 \\
\hline
\end{tabular}

By their shape and paste age they may be assimilated to Ettringite [23,30,31].

Smaller agglomerates have a micronic size and are constituted of few sub-micronic entities like the cubic hydration products or the modular and angular particles resulting from attrition (Fig. 5a and 5b). Interactions between sub-micronic particles and micronic particles with particles of several tens or even hundreds of microns are always present (Fig. 5c). There are also agglomerates constituted of unit particles of ten microns. However, it is difficult to determine agglomeration or aggregation (chemical bonds between particles) is involved because the cohesion between particles appears to be strong (Fig. 5d).

SEM snapshots of the paste diluted in ethyl alcohol display the presence of many acicular sub-micronic particles that correspond to the appearance of new populations with a mode located at $0.3 \mu \mathrm{m}$. Furthermore, cubic crystals are present in greater numbers and small agglomerates are almost non-existent in the diluted area (Fig. 4c). However, microscopic observations show the presence of agglomerates of several hundred microns in the concentrated area (Fig. 4d), that do not show by laser granulometry in this medium. These agglomerates are constituted by particles of the order of several to $10 \mu \mathrm{m}$.

This comparative analytical approach using laser granulometry and SEM allowed to identify and differentiate the different particle populations, agglomerated or not. The results are summarized in Fig. 3.

\subsection{Influence of the superplasticizers on dispersion state}

It is possible to define the granular populations influenced by the addition of a superplasticizer by comparing the particle size distributions of an admixtured paste analyzed in deionized water and the reference distribution chosen on the basis of the previous results; namely, the unadmixtured cement paste analyszed in deionized water (agglomerant medium) which shows the more important spread size (93 $\mu \mathrm{m})$. Indeed, the addition of polycarboxylate causes a shift of the particle size distribution toward smaller diameters (mode evolves from $25 \mu \mathrm{m}$ to around $20 \mu \mathrm{m}$ ). This influence is shown in Fig. 6.

As a first step, the presence of two singular points, noted $T_{D}$ and $T_{A}$ ( $\mathrm{D}$ for dispersion and $\mathrm{A}$ for agglomeration) can be noticed and correspond to the intersections of the two distributions. These points, like isobestic points, highlight mass transfers between the different areas they enclose [26]. The first area, noted $\mathrm{A}_{4}$, corresponds to the granular population that disappears under the influence of the superplasticizer; it thus characterizes dispersed agglomerates. A part of this dispersed population, observed by microscopy (Fig. 7), moves to the area noted $A_{2}$. However, for some formulations, the presence of an area, noted $A_{3}$, the mode of which is located near $150 \mu \mathrm{m}$, can be observed. Its origin may be twofold: either it comes mainly from some unit particles that were not ground down by attrition, initially present in the cement and sampled randomly (do not forget these distributions are expressed in volume which strongly overestimates the largest particles, especially
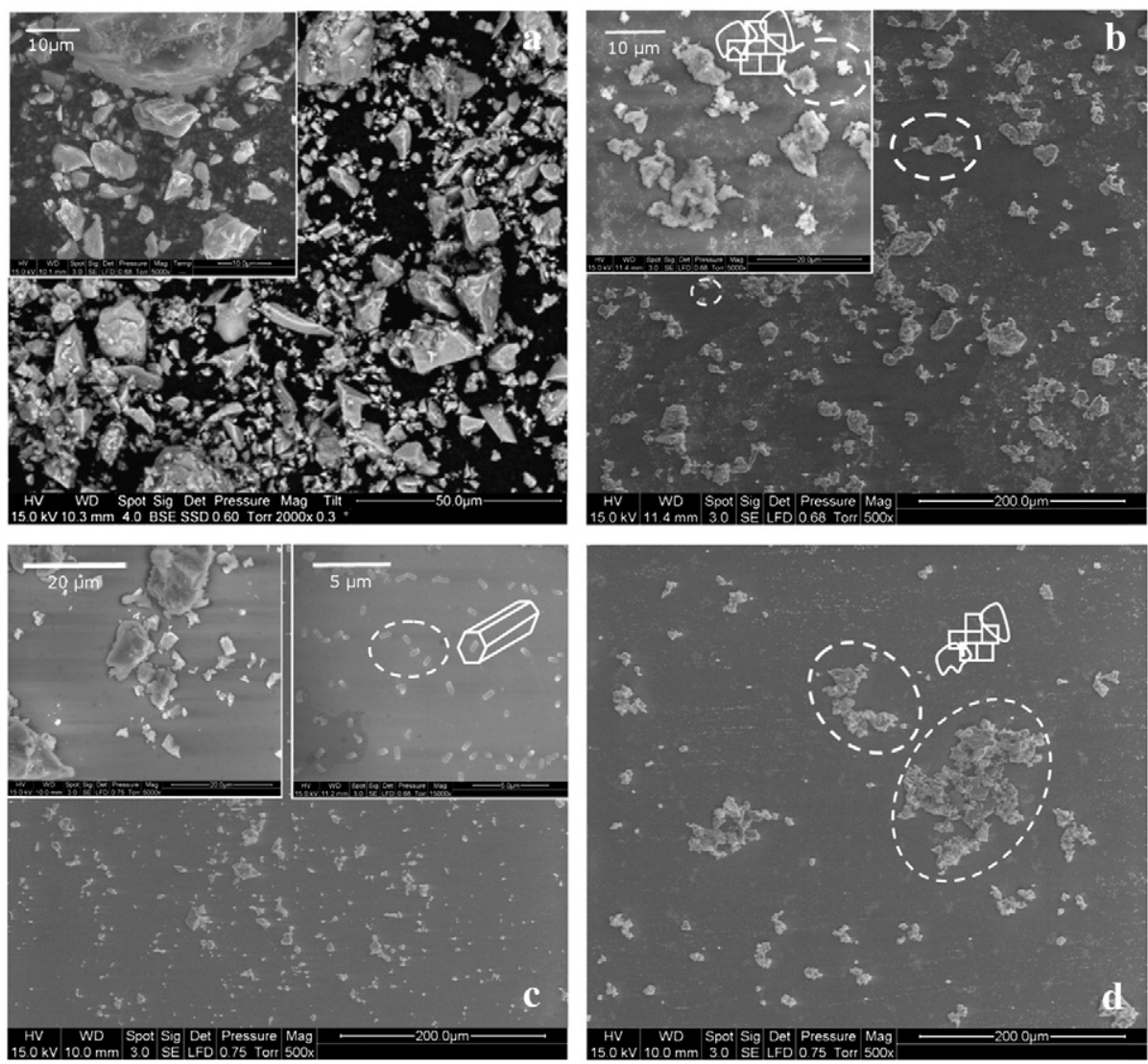

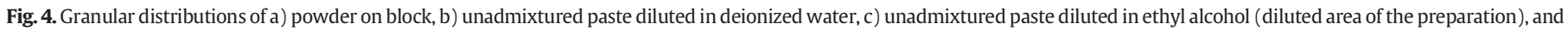
d) unadmixtured paste diluted in ethyl alcohol (concentrated area). 

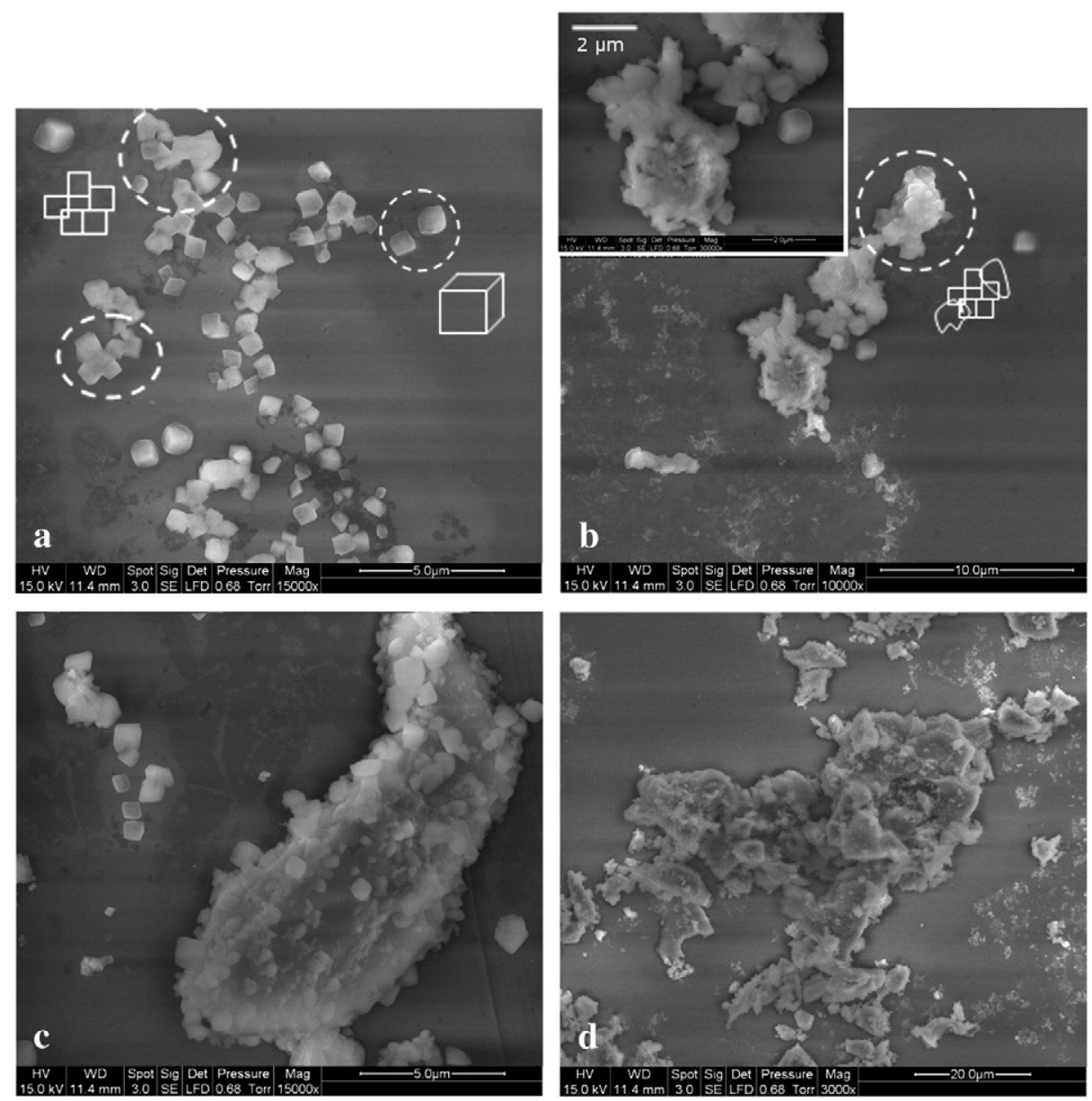

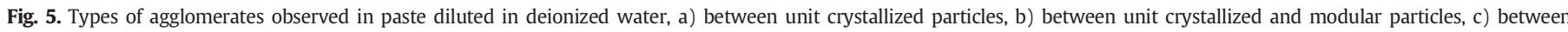
sub-micronic particles on a particle of the mode, and d) strong agglomeration of micronic particles.

with a logarithmic abscissa scale), or it comes from a phenomenon of reagglomeration. Because of the sampling method (mid-height and equal distance of the side and the middle of the beaker) and the presence of this population when the sample is first sieved to $63 \mu \mathrm{m}$, it is possible to assert that it is mainly constituted by agglomerates and not unit particles unaffected by attrition. However, it is difficult to determine the size fractions which constitute them because these agglomerates were not observed by microscopy.

It is necessary to integrate the probability of re-agglomeration phenomena in the influence of superplasticizer. Indeed, just a low proportion can impact the particle size distribution because of the overestimation of the largest particles, and thus may influence the dispersion index.

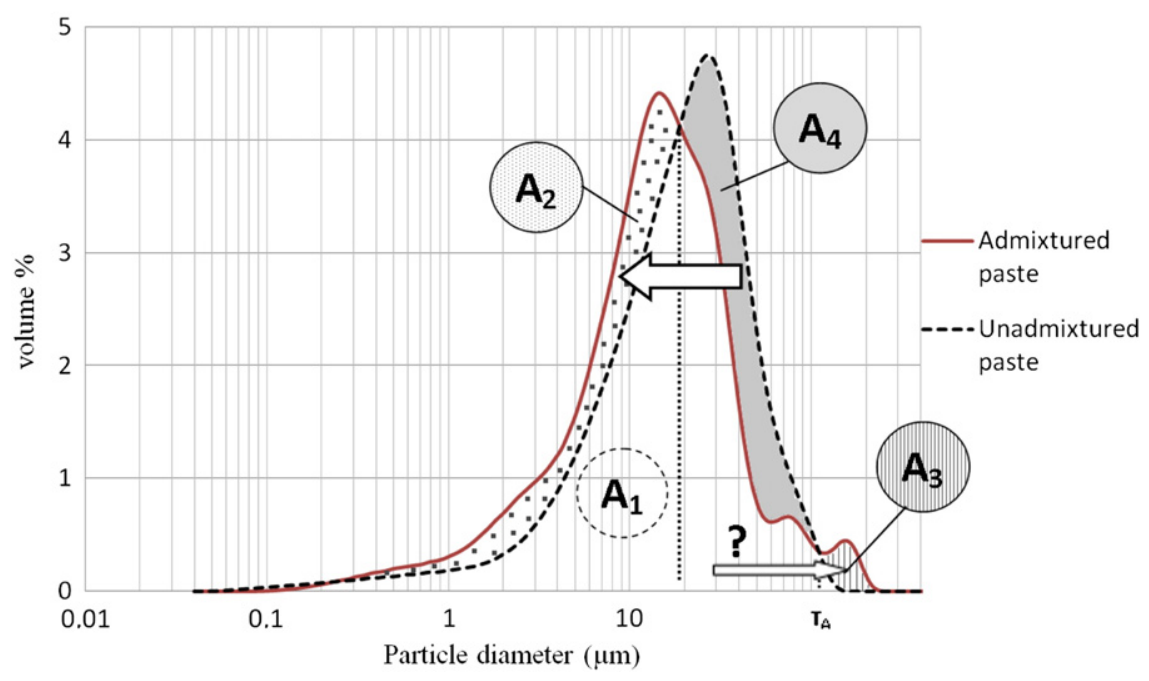

Fig. 6. Identification of dispersion phenomena caused by the addition of $0.1 \%$ of polycarboxylate $A$. 


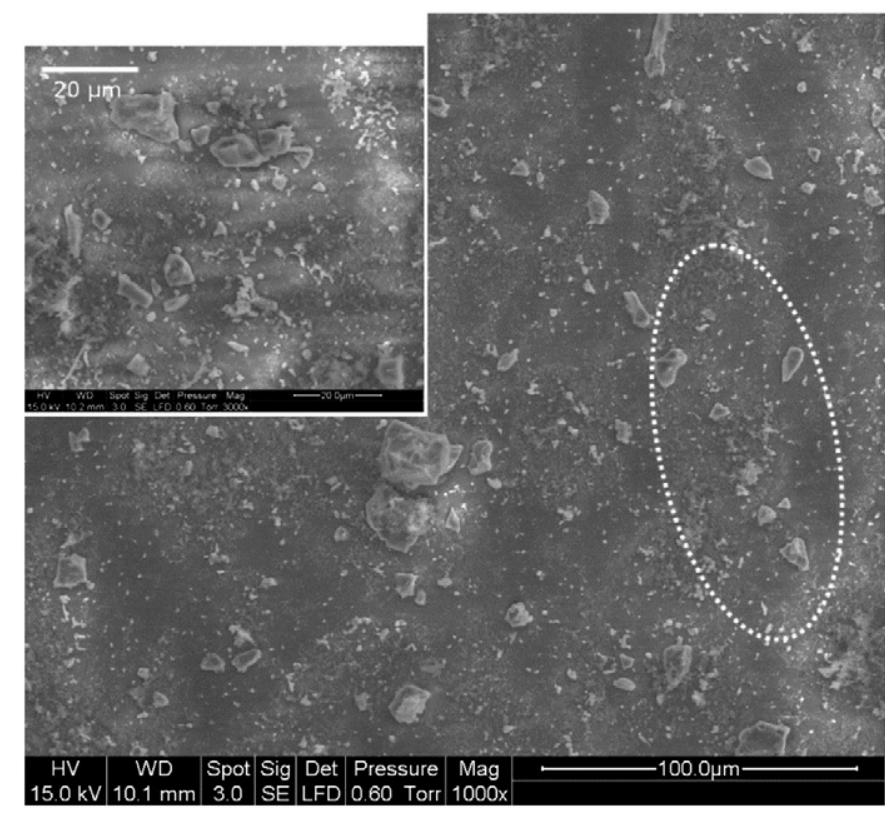

Fig. 7. SEM micrograph of an admixtured cement paste with $1 \%$ of polycarboxylate A.

Therefore, two indices were determined in order to characterize the mesostructural evolutions, on the one hand, the phenomenon of true dispersion, and on the over hand, the overall influence of the polycarboxylate (integrating dispersion and agglomeration phenomena). The evolution of the first one, characterizes the dispersion of the particles and more accurately the organization of the system at the mesoscopic scale. Furthermore, the comparison between the two indices allows determining the influence of re-agglomeration and having hindsight with the dispersion index.

The first index denoted $I_{D}$ was developed in order to consider only the proportion of really dispersed particles. It is defined as follows:

$\operatorname{ID}(\%)=\left(\mathrm{As}_{<\mathrm{TD}}-\mathrm{Aref}_{<\mathrm{TD}}\right) * 100 / \mathrm{A}_{1}=\mathrm{A}_{2} * 100 / \mathrm{A}_{1}=\mathrm{A}_{2}$

where $A s_{<T D}$ is the area lower than $T_{D}$ located under the curve of the sample.

Aref $_{\angle \mathrm{TD}}$ is the area lower than $\mathrm{T}_{\mathrm{D}}$ located under the curve of the reference.

$A_{1}$ is the total area located under the curve of the reference $(=100 \%$ because the curves are normalized).

$A_{2}$ is the area located between the curve of the sample and the curve of the reference under the transfer point $T_{D}$.

A second index, denoted $\mathrm{I}_{\mathrm{DG}}$, was proposed to represent the proportion of particles influenced by the addition of polycarboxylate. Hence, it includes both dispersion and re-agglomeration phenomena. By comparison with $I_{D}$, this index allows to highlight the influence of the reagglomeration phenomenon and thus stand back with the interpretation of the $I_{D}$. It is defined as follows:

$\mathrm{ID}(\%)=\left(\operatorname{Aref}_{\mathrm{TD}-\mathrm{TA}}-\mathrm{As}_{\mathrm{TD}-\mathrm{TA}}\right) * 100 / \mathrm{A}_{1}=\mathrm{A}_{4} * 100 / \mathrm{A}_{1}=\mathrm{A}_{4}$

where Aref $f_{T D-T A}$ is the area between $T_{D}$ and $T_{A}$ located under the curve of the reference.

$\mathrm{As}_{\mathrm{TD}-\mathrm{TA}}$ is the area between $\mathrm{T}_{\mathrm{D}}$ and $\mathrm{T}_{\mathrm{A}}$ located under the curve of the sample.

$\mathrm{A}_{4}$ is the area located between the curve of the sample and the curve of the reference between $T_{D}$ and $T_{A}$ (equal to Aref $\mathrm{TD}_{\mathrm{TD}-\mathrm{TA}}-\mathrm{As}_{\mathrm{TD}-\mathrm{TA}}$ ), $\mathrm{A}_{4}=\mathrm{A}_{2}+\mathrm{A}_{3}$.

$A_{3}$ is the area located between the curve of the sample and the curve of the reference above $T_{A}$.

Calculated areas correspond to the sum of the volume percentages of the different size fractions contained within the considered range.

Cement paste is a heterogeneous material, so for each formulation the analysis was performed on five different samples in order to take into account the variability of the sampling. The average values of the two indices and their associated standard deviations obtained for each formulation are shown in Fig. 8.

According to the Fig. 8, the two indices show similar variations and values. So, the influence of re-agglomeration on the dispersion index is negligible and is not dependent on the amount of polycarboxylate. The evolution of the $I_{D}$ is discriminating depending on the amount of superplasticizer. The PCE AA presents other trends than the others with present close values. Indeed, in the case of the last three, the $I_{D}$ value increases with dosage up to 0.08 wt.\% of polycarboxylate, characterizing an increasing dispersion of the particles. Beyond this dosage, the $I_{D}$ value is stable or decreases, characterizing the re-agglomeration phenomenon on the size range lower than $100 \mu \mathrm{m}$. So, the optimal dispersion is observed at $0.08 \%$ and the PCEs A and B seem to be generally the more effective but the standard deviation is too important to make a real difference. The slight tendency that $\mathrm{I}_{\mathrm{D}}$ and $\mathrm{I}_{\mathrm{DG}}$ differ at $1 \mathrm{wt} . \%$ underlines a started re-agglomeration phenomenon above $100 \mu \mathrm{m}$, but this phenomenon is negligible compared to the reagglomeration in the range below $100 \mu \mathrm{m}$. In the case of the PCE AA, the $\mathrm{I}_{\mathrm{D}}$ seems stable up to $0.1 \%$ and then increases, traducing a poor dispersion of particles at low dosages. Therefore, beyond a given ester grafting ratio (between $\mathrm{AA}$ and $\mathrm{A}$ ), this parameter is not a decisive structural parameter on the efficiency of polycarboxylates.

\subsection{Comparison with the rheological behavior}

The rheograms obtained for different amounts of polycarboxylate B are shown in Fig. 9. The reproducibility is acceptable for all the amounts tested. For a same shear rate, the measured stress decreases when the amount of polycarboxylate increases, suggesting an increase of the
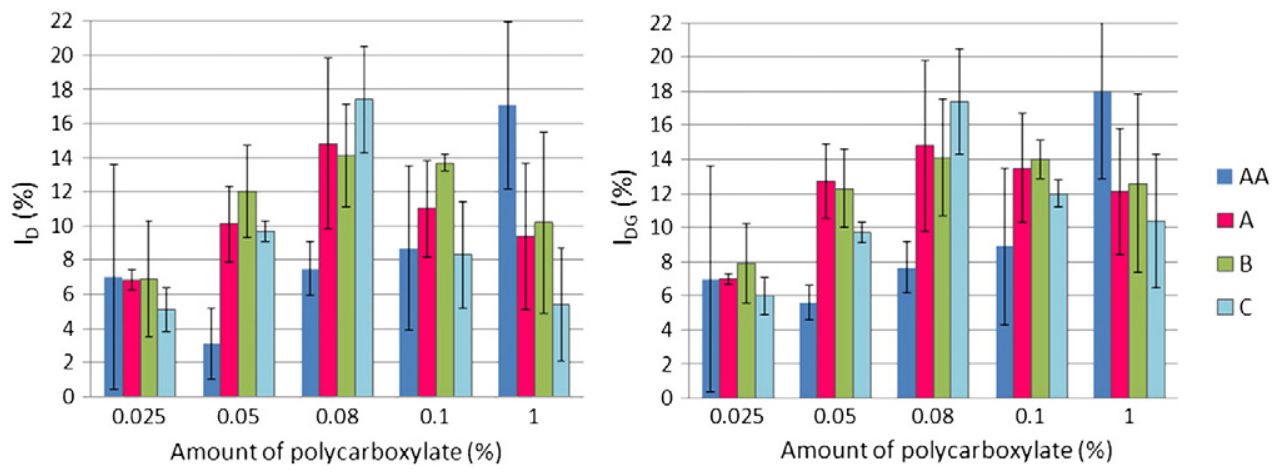

Fig. 8. $I_{D G}$ and $I_{D}$ indices obtained for the different formulations containing the polycarboxylates $A A, A, B$ and $C$. 


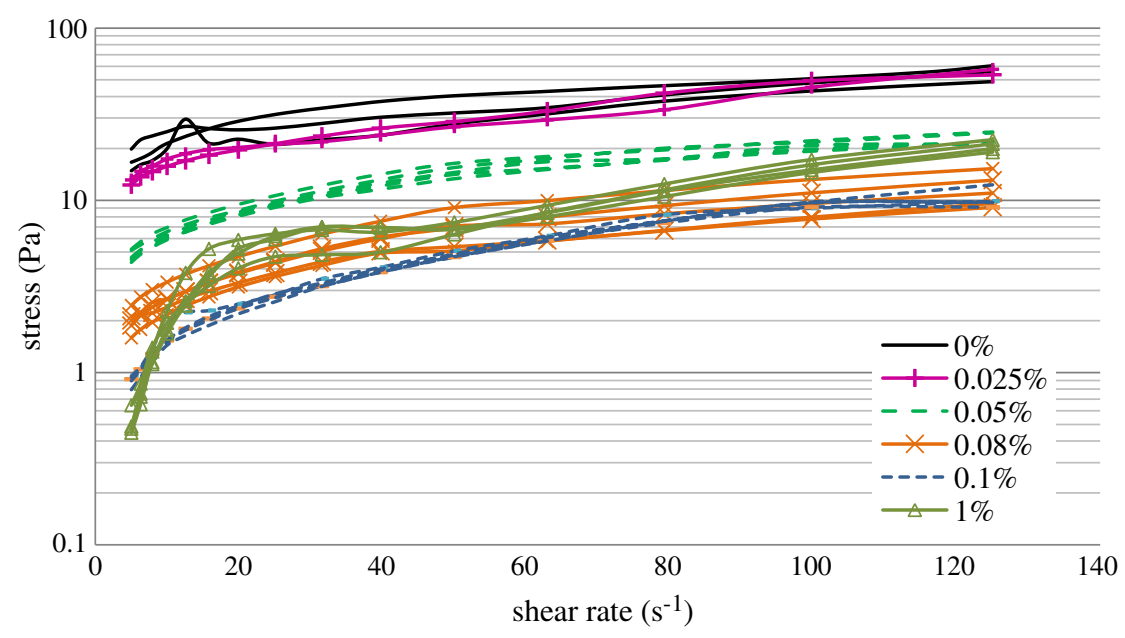

Fig. 9. Rheograms obtained for the different amounts of polycarboxylate B tested.

paste fluidity. This influence is characterized by the evolution of the yield stress according to the Bingham model applied between $30 \mathrm{~s}^{-1}$ and $125 \mathrm{~s}^{-1}$. This model takes into account the lowest stress necessary to cause the flow of the material, called yield stress and noted $\tau_{0}$. Beyond this value, there is a linear relationship between the shear rate and the yield stress. It is reminded that at this stage of hydration (dormant period) the reactivity is slow down. So, the modifications of the rheological behavior induced by formation of hydrates are negligible [31,32]. In the same way as for the dispersion indices, each formulation was tested on five different samples and the values shown in Fig. 10 represent average values and the standard deviations associated.

According to the $\mathrm{I}_{\mathrm{D}}$ values, the PCEs A, B and C present an important decrease of the yield stress when the amount of admixture increases up to 0.08 wt.\% even at low dosages (indeed the yield stress value of the unadmixtured paste is about $30 \mathrm{~Pa}$. while it is lower than $15 \mathrm{~Pa}$ for admixtured pastes). Beyond $0.08 \%$, the yield stress continues to decrease whereas the dispersion index decreases. This can be explained by the fact that the particles in agglomerates formed in excess of polycarboxylate are linked together by weaker physical bonds than in a unadmixtured paste. So, they do not impact the rheological behavior. In the case of the PCE AA, the yield stress first increases up to $0.1 \%$ by unexplained way, and then decreases strongly at $1 \%$. This behavior may be related to the $I_{D}$ which behavioral change was observed at the same dosage. The comparison between the established indices and the flow behavior of the different formulations allowed reaching a better understanding of the real influence of superplasticizers in the dispersion state, and thus the mesostructural organization of the cement pastes.

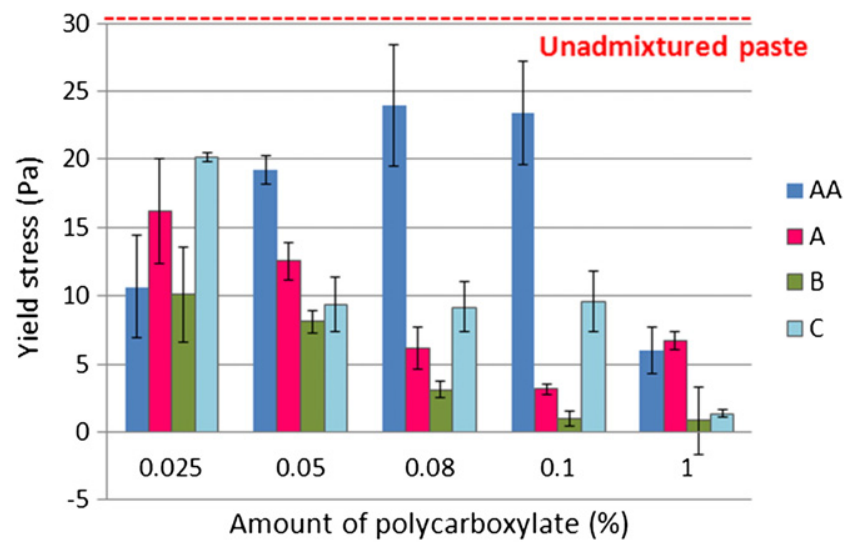

Fig. 10. Rheological behavior of cement pastes.
We can notice that, contrary to the dispersion index, the measure of the rheological behavior allows to differentiate the polycarboxylates $\mathrm{A}$, $B$ and $C$. Indeed, the polycarboxylate B presents the best efficiency for all the formulations. This result is probably due to an optimized ester ratio in the chemical structure of the polycarboxylate [27-29]. Indeed, beyond this ratio, we assume that the amount of carboxylate groups is not sufficient to allow a sufficient adsorption, or an excess of PEO groups in the molecular structure may favor bridging phenomena between particles, thus causes re-agglomeration phenomena. That explains the increase of the yield stress between the polycarboxylates B and C. In the case of the PCE AA, it is possible to assume that the ester grafting ratio is too low to allow a sufficient steric repulsion between particles. Note that even if the differences are greater than the standard deviation associated to these values, it is not an important difference.

\section{Conclusion}

In this study, the dispersion state of cement pastes was characterized by using a morpho-granulometric approach based on the complementarity between scanning electron microscopy and laser granulometry. Different granular populations, agglomerates or unit particles, were identified. It was possible to distinguish particles ground by attrition during batching from crystallized hydrated particles. Two agglomeration mechanisms were observed: the first one arising from (sub) micronic particle agglomeration upon bigger particles of several tens of microns; the second one resulting from the agglomeration of (sub) micronic particles upon themselves. Most of the agglomerates formed are supracolloids.

As a second step, several dispersion states were generated by addition of superplasticizer with different dosages or ratio of ester groups. Two indices were developed upon area differences under the particle size distributions curves. The first one, called $\mathrm{I}_{\mathrm{D}}$ characterizes the proportion of truly dispersed particles, and give directly information about the dispersion state of the paste. The second index called $\mathrm{I}_{\mathrm{DG}}$ characterizes the overall influence of the polycarboxylate, including dispersion and possible re-agglomeration phenomena beyond $100 \mu \mathrm{m}$ that can influence the $I_{D}$ index. So, by comparison between the two indices, it is possible to evaluate the importance of theses agglomerates higher than $100 \mu \mathrm{m}$.

The defined dispersion indices allow characterizing the evolution of mesostructure and having a better understanding of the evolution of the rheological behavior with the dosage of superplasticizer. But in these studied cases, they are not accurate enough to differentiate the influence of a change in the molecular structure of the polycarboxylatebased superplasticizer. 
However this morpho-granulometric approach in comparison with the rheological behavior of the cement paste may be easily transferred to other studies characterizing the influence of surfactants on the dispersion state of different types of suspensions.

\section{Acknowledgments}

The authors wish to thank Mr. Frederic Leising and Mr. Pascal Boustingorry (CHRYSO) for their collaboration in the project.

\section{References}

[1] J.P. Ollivier, A. Vichot, La durabilité des bétons: bases scientifiques pour la formulation des bétons, Presse de l'Ecole nationale des ponts et chausses, 2008.

[2] P. Coussot, C. Ancey, Rhéophysique des pâtes et des suspensions, EDP Sciences, 1999.

[3] A. Zingg, F. Winnefeld, L. Holzer, J. Pakusch, S. Becker, L. Gauckler, Adsorption of polyelectrolytes and its influence on the rheology, zeta potential, and microstructure of various cement and hydrate phases, J. Colloid Interface Sci. 323 (2) (2008) 301-312.

[4] F. Perche, Adsorption de polycarboxylates et de lignosulfonates sur poudre modèle et ciments, Ecole polytechnique fédérale de Lausanne, 2004.

[5] J. Plank, C. Hirsch, Impact of zeta potential of early cement hydration phases on superplasticizer adsorption, Cem. Concr. Res. 37 (4) (2007) 537-542.

[6] S. Srinivasan, S.A. Barbhuiya, D. Charan, S.P. Pandey, Characterising cementsuperplasticizer interaction using zeta potential measurements, Constr. Build. Mater. 24 (12) (2010) 2517-2521.

[7] A. Zingg, F. Winnefeld, L. Holzer, J. Pakusch, S. Becker, R. Figi, L. Gauckler, Interaction of polycarboxylate-based superplasticizer with cement containing different C3A amounts, Cem. Concr. Compos. 31 (3) (2009) 153-162.

[8] M. Palacios, Y.F. Houst, P. Bowen, F. Puertas, Adsorption of superplasticizer admixtures on alkali-activated slag pastes, Cem. Concr. Res. 39 (8) (2009) 670-677.

[9] L. Ferrari, J. Kaufmann, F. Winnefeld, J. Plank, Interaction of cement model systems with superplasticizers investigated by atomic force microscopy, zeta potential, and adsorption measurements, J. Colloid Interface Sci. 347 (1) (2010) 15-24.

[10] K. Yoshioka, Adsorption characteristics of superplasticizers on cement component minerals, Cem. Concr. Res. 32 (10) (2002) 1507-1513.

[11] D.P. Bentz, E.J. Garboczi, C.J. Haecker, O.M. Jensen, Effects of cement particle size distribution on performance properties of Portland cement based-materials, Cem Concr. Res. 29 (10) (1999) 1663-1671.

[12] J.J. Thomas, J.J. Biernacki, J.W. Bullard, S. Bishnoi, J.S. Dolado, G.W. Scherer, A. Luttge, Modeling and simulation of cement hydration kinetics and microstructure development, Cem. Concr. Res. 41 (2011) 1257-1278.

[13] H. Binici, O. Aksogan, I.H. Cagatay, M. Tokyay, E. Emsen, The effect of particle size distribution on the properties of blended cements incorporating GGBFS and natural pozzolan (NP), Powder Technol. 177 (2007) 140-147.
[14] W. vom Berg Influence of specific surface and concentration of solids upon the flow behavior of cement pastes, Mag. Concr. Res. 31 (109) (1979) 211-216.

[15] S. Tadier, N. Le Bolay, C. Rey, C. Combes, Co-grinding significance for calcium carbonate-calcium phosphate mixed cement. Part I: effect of particle size and mixing on solid phase reactivity, Acta Biomater. 7 (4) (2011) 1817-1826.

[16] S. Mansoutre, Water retention and granular rheological behavior of fresh C3S paste as a function of concentration, Cem. Concr. Res. 29 (9) (1999) 1441-1453.

[17] T. Zhang, Q. Yu, J. Wei, P. Zhang, A new gap-graded particle size distribution and resulting consequences on properties of blended cement, Cem. Concr. Compos. 33 (2011) 543-550

[18] A.P. Silva, A.M. Segadães, D.G. Pinto, L.A. Oliveira, T.C. Devezas, Effect of particle size distribution and calcium aluminate cement on the rheological behaviour of all-alumina refractory castables, Powder Technol. 226 (2012) 107-113.

[19] B. Felekoglu, Effects of PSD and surface morphology of micro-aggregates on admixture requirement and mechanical performance of micro-concrete, Cem. Concr. Compos. 29 (2007) 481-489.

[20] B. Felekoglu, A new approach to the characterisation of particle shape and surface properties of powders employed in concrete industry, Constr. Build. Mater. 23 (2009) 1154-1162.

[21] V. Lilkov, E. Dimitrova, S. Gaidardzhiev, Microscopic and laser granulometric analyses of hydrating cement suspension, Cem. Concr. Res. 29 (1999) 3-8.

[22] S. Paumier, A. Pantet, P. Monnet, Evaluation of the organization of the homoionic smectite layers $(\mathrm{Na}(+)$ or $\mathrm{Ca}(2+))$ in diluted dispersions using granulometry, microscopy and rheometry, Adv. Colloid Interf. Sci. 141 (1-2) (2008) 66-75.

[23] A. Zingg, L. Holzer, A. Kaech, F. Winnefeld, J. Pakusch, S. Becker, L. Gauckler, The microstructure of dispersed and non-dispersed fresh cement pastes - new insight by cryo-microscopy, Cem. Concr. Res. 38 (2008) 522-529.

[24] J. Stark, Recent advances in the field of cement hydration and microstructure analysis, Cem. Concr. Res. 41 (2011) 666-678.

[25] S. Maximilien, Study of the reactivity of clinkers by means of the conductometric test, Cem. Concr. Res. 27 (1) (1997) 63-73.

[26] N. Azema, M.F. Pouet, C. Berho, O. Thomas, Wastewater suspended solids study by optical methods, Colloids Surf. A 204 (1-3) (2002) 131-140.

[27] J.Y. Shin, J.S. Hong, J.K. Suh, Y.S. Lee, Effects of polycarboxylate-type superplasticizer on fluidity and hydration behavior of cement paste, Korean J. Chem. Eng. 25 (6) (2008) 1553-1561.

[28] C. Li, N. Feng, Y. Li, R. Chen, Effects of polyethylene oxide chains on the performance of polycarboxylate-type water-reducers, Cem. Concr. Res. 35 (5) (2005) 867-873.

[29] K. Yamada, Effects of the chemical structure on the properties of polycarboxylate-type superplasticizer, Cem. Concr. Res. 30 (2) (2000) 197-207.

[30] J.W. Bullard, H.M. Jennings, R.A. Livingston, A. Nonat, G.W. Scherer, J.S. Schweitzer, K.L. Scrivener, J.J. Thomas, Mechanisms of cement hydration, Cem. Concr. Res. 41 (2001) 1208-1223.

[31] C. Rößler, A. Eberhardt, H. Kučerová, B. Möser, Influence of hydration on the fluidity of normal Portland cement pastes, Cem. Concr. Res. 38 (7) (2008) 897-906.

[32] G. Sant, C.F. Ferraris, J. Weiss, Rheological properties of cement pastes: a discussion of structure formation and mechanical property development, Cem. Concr. Res. 38 (7) (2008) 1286-1296. 